\title{
Cryptographic Protocols to Fight Sinkhole Attacks on Tree-based Routing in Wireless Sensor Networks
}

\author{
Anthonis Papadimitriou \\ INRIA-Saclay \\ anthony@di.uoa.gr
}

\author{
Fabrice Le Fessant \\ INRIA-Saclay \\ fabrice.le_fessant@inria.fr
}

\author{
Aline Carneiro Viana \\ INRIA-Saclay \\ aline.viana@inria.fr
}

\author{
Cigdem Sengul \\ DT Labs, TU-Berlin \\ cigdem.sengul@telekom.de
}

\begin{abstract}
This work introduces two new cryptographic protocols of different complexity and strength in limiting network degradation caused by sinkhole attacks on tree-based routing topologies in Wireless Sensor Networks (WSNs). The main goal of both protocols is to provide continuous operation by improving resilience against, rather than detection of, these attacks. Building up resilience may prove to be more beneficial because it allows operating (or graceful degradation) in the presence of attacks. Furthermore, while resilience mechanisms do not dismiss detection mechanisms, detection mechanisms often introduce more complexity and so, more weaknesses to the system, which might not justify their benefits. We provide a simulation study of the two protocols for three different routing strategies, that encompass typical routing strategies used in WSN. The results of our simulation study show that our cryptographic protocols are effective in improving resilience against sinkhole attacks, even in the presence of some collusion.
\end{abstract}

\section{INTRODUCTION}

Wireless Sensor Networks (WSN) are penetrating more and more in our daily life. As a consequence, security has become an important matter for these networks. Indeed, many WSN protocols are not secured even against simple attacks. Let's take a simple scenario for a possible attack: a supermarket is protected during closing hours by a WSN, where each sensor is able to sense any movement in its neighbourhood, and report an alarm to the sink at the center of the supermarket. A thief can put a sensor in the neighbourhood of the supermarket, that tells other sensors within the supermarket that it is closer to the sink (i.e., sinkhole attack). All their messages for the sink, especially alarms, will be routed through that sensor, that will be able to discard alarm messages (i.e., selective forwarding attack) and let the thief enter the supermarket.

Recent work [1], [2] has mostly focused on how such an attack can be detected with high probability. However, attack detection often increases the complexity of the system and thus, increases the possibility of other attacks. Furthermore, fault-detection mechanisms or network debuggers can also be effected by sinkhole attacks [3]. In [3], a network debugger is proposed, which requires periodic metric collection at the sink. The sink decides which nodes have delivered sufficient data and if not, infers the causes of the failures. However, during their evaluations, the authors ran into a problem : some nodes had a route to a node that was not the sink. The authors validated that the network was exposed temporarily to an extraneous node with the same ID as a node already in the network. That node advertized itself as a sink, confusing the network. From that point on, due to a bug in the routing code, the network never recovered. This example shows that ensuring built-in resilience to sink-hole attacks would be more effective. Consequently, we propose two RESIlient and Simple Topology-based reconfiguration protocols: RESIST-1 and RESIST-0. RESIST-1 prevents a malicious node from modifying its advertised distance to the sink by more than one hop, while RESIST-0 does not allow such lying at the cost of additional complexity.

We studied the performance of RESIST-1 and RESIST-0 through simulations, for three tree-based routing protocols that encompass different routing strategies. Our simulation results show that our reconfiguration protocols are effective in improving resilience against sinkhole attacks in WSNs. Finally, we conclude with a discussion on implementation issues, such as the use of cryptography in sensor networks, and on the impact of collusion on our protocols.

The remainder of this paper is structured as follows. In Section II we present the system model. In Section III, we lay out our proposal for two simple and resilient topologybased routing protocols. Performance results are presented in Section IV and discussions in Section V. The Section VI overviews the current literature. Finally, Section VII concludes with future work.

\section{Problem StATEMEnT}

This section presents the network and the threat models, as well as, the attack metric, the Risk Factor, used in our simulations.

\section{A. Network model}

We consider a connected WSN consisting of $N$ static sensors randomly scattered on a geographical area, and only one sink, each with unique IDs. All sensors are identical in terms of computational, memory, and communication capabilities. In order to keep the focus on the resilience analysis of our protocols we leave the evaluation of energy consumption for future work. Nodes do not have access to location information. Each node or the sink is able to communicate wirelessly with a subset of nodes (its neighbors) that are in its transmission range, $r_{t}$. We consider a large set of routing protocols relying on tree-based topology construction [4]-[10]. The data is thus, routed from sensor nodes to the sink through a tree rooted at the sink. The routing tree is an aggregation of the shortest paths from each sensor to the sink based on a cost metric, which can represent different application requirements (e.g., 
cost hop count, loss, delay). In this work, the routing tree is built by using the hop distance to the sink.

\section{B. Threat model}

We focus on sinkhole attacks launched by compromised nodes inside the network. In our threat model, sensors cannot lie about their identities due to the presence of cryptographic measures [11]. In general, we assume malicious sensors are not colluding (i.e., collaborating to increase the impact of the attack). The impact of collusion is discussed in Section V.

We assume that public-key cryptographic primitives are available on all sensors (refer to [12] for a survey on key distribution in WSNs). Furthermore, recently it has also been shown that public key infrastructure is viable for WSNs [13]. Hence, in our model, all sensors only know and trust the public key $K_{p u b}^{\text {sink }}$ of the sink. Additionally, each sensor $X$ has a pair of public-private keys $\left(K_{p u b}^{X}, K_{p r i}^{X}\right)$ that it can use to prove its identity. These key pairs can be generated and uploaded offline to the sensors before the deployment. Using these key pairs, nodes perform authentication and sign data messages.

\section{Risk model}

Many papers in the literature measure the strength of an attack by the number of compromised nodes. This number is misleading, as, for example, a few well-positioned compromised nodes may have a much larger impact than many compromised nodes that are positioned badly in the network.

Consequently, in this paper, we use a new metric, called Risk Factor [14] to measure the strength of an attack. Intuitively, the Risk Factor is the probability that a message reaches compromised nodes (that might drop it) on its route to the sink, and inherently captures parameters such as: the number of compromised sensors, their position, the density, and the size of the network. It is computed iteratively starting from the sink using the following formula:

$$
\begin{aligned}
& \text { NodeRisk }_{X}= \begin{cases}0 & \text { if } X \text { is the sink } \\
p & \text { if } X \text { is malicious } \\
\frac{\sum_{Y \in N_{X} \mid d_{Y}<d_{X} N o d^{\prime} R \text { Risk }}}{\left\|\left\{Y \in N_{X} \mid d_{Y}<d_{X}\right\}\right\|} & \text { otherwise, }\end{cases} \\
& \text { RiskFactor }=\frac{\sum_{X \in V} \text { NodeRisk }_{X}}{\|V\|}
\end{aligned}
$$

We evaluated Risk Factor with different number of compromised nodes using 3 different positioning strategies: (1) ring placement at a distance equal to the transmission range of the sink (denoted as $R$ ), (2) ring placement at a distance $1.5 R$ and (3) random placement. Fig. 1 illustrates the representativeness of the Risk factor compared to "the number of compromised nodes" For instance, the Risk Factor is able to capture that surrounding the sink at a closer distance performs better than random placement. Note that using "the number of compromised nodes" the risk would increase regardless of how these nodes are positioned. Furthermore, the majority of the time, the risk increases more slowly or remains constant after a constant amount of compromised nodes.

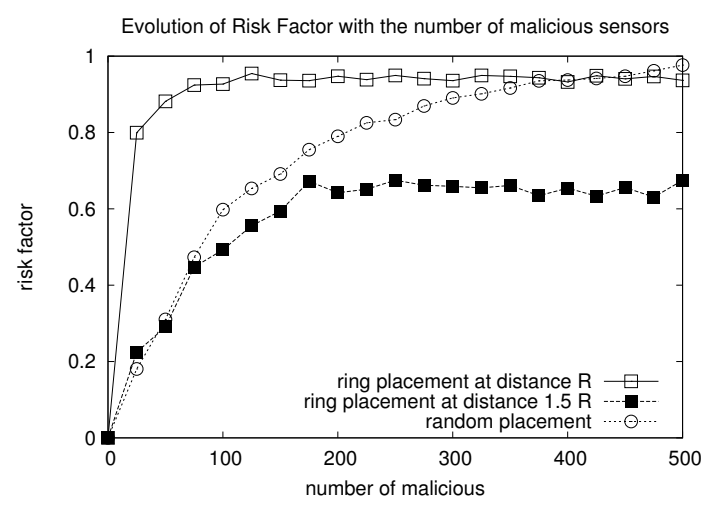

Fig. 1. The Risk Factor grows very fast with the first compromised nodes, and more slowly afterwards. A few well-positioned nodes (a ring at distance 1) can impact the network as much as hundreds of nodes randomly positioned.

\section{SECURITY PROTOCOLS}

To achieve higher resilience in tree-based routing protocols [4]-[10], we propose two protocols. These protocols are used at the tree reconfiguration phase triggered by the sink. Note that we do not have special constraints on the period between reconfigurations: it can be chosen either similar to the current tree-based routing protocols, based on cost, or topology vulnerability. We define a class of RESIST- $h$ reconfiguration protocols that allow malicious nodes to modify their advertised distance to the sink, but no more than $h$ hops. Based on this definition, we introduce two protocols, RESIST-1 and RESIST0 , which are presented in the remainder of this section. We also describe here cryptographic operations and message contents of the proposed protocols, but refer the reader to Section V for an efficient way of implementing them.

\section{A. Simple reconfiguration protocol (RESIST-1)}

The reconfiguration starts by the sink sending a Hello(epoch,tokens) message to all its neighbors, where epoch is a strictly increasing timestamp, chosen by the sink and tokens is a list of tokens $\left[T_{0}, T_{1}, T_{2}, \ldots, T_{R}\right]$. Essentially, each token is a ( $k$, epoch) pair signed by the sink, where $k$ is the token number:

$$
T_{k}=(k, \text { epoch })_{\text {signed }\left(K_{p r i}^{\text {sink }}\right)}
$$

When a sensor receives a Hello message, and after verifying that the tokens are correctly signed by the sink (i.e. verified by using the public key $K_{p u b}^{\text {sink }}$ ), it does the following:

- If the epoch is new, it remembers the identity of the node sending it (i.e., his parent), and propagates the Hello message after removing the smallest token from the list of tokens. In other words, it receives Hello (epoch, $\left.\left[\mathbf{T}_{\mathbf{k}}, T_{k+1}, \ldots, T_{R}\right]\right)$ but sends Hello (epoch, $\left.\left[\mathbf{T}_{\mathbf{k}+\mathbf{1}}, \ldots, T_{R}\right]\right)$.

- If the epoch is already known, but the Hello message advertises a shorter hop distance to the sink (i.e., contains a smaller token), the node might follow different approaches. A selfish approach would only update the node itself, while a gossip approach would also propagate 
a new Hello message to the neighbors. In the rest of the paper, we follow the gossip approach.

Each sensor remembers as its parent the sensor that sent it the smallest token signed by the sink for the most recent epoch. Note that the token number of the smallest token is also the hop distance to the sink. Alternatively, sensors can also remember all the nodes that advertise the shortest distance for a given epoch. In Section IV, we also evaluate this approach. Sinkhole attack resilience: A compromised node can directly forward a Hello message without dropping the first token. Assume that the node is the first compromised node on the branch that the Hello message travels. Then, if the compromised node is at distance $k$ from the sink, its neighbors would believe they are at distance $k$ too, and so they would believe that the compromised node is at distance $k-1$. Nevertheless, the compromised node cannot pretend to be at a distance smaller than $k-1$, because it would be unable to provide smaller tokens than $T_{k}$. Note that as the Hello message travels down the tree, it might encounter other malicious nodes that do not drop the token before forwarding the message. In this case, each uncompromised sensor would believe to be at a shorter distance to the sink depending on how many malicious nodes exist before it (e.g., if the number of malicious nodes between the sensor and the sink is 2 , then it will at most believe it is 2 hops closer to the sink than the reality). Even if we do not have a strong bound on the deviation from the real distance in RESIST-1 (i.e., it increases with the number of malicious nodes on the path), this might not degrade the performance significantly because the main impact is caused by the malicious node closest to the sink.

\section{B. Complex reconfiguration protocol (RESIST-0)}

To provide higher resilience, we next propose a more complex reconfiguration protocol (RESIST-0). This protocol is inspired by a protocol used to measure availability in peerto-peer networks [15], where newly generated pairs of cryptographic keys are diffused in the network at every round. The sink sends a Hello(epoch, $\left.\left[T_{0}, T_{1}, \ldots, T_{R}\right]\right)$ message, where the generated tokens are:

$$
T_{k}=\left(\left(k, \text { epoch }, K_{\text {pub }}^{k}\right)_{\text {signed }\left(K_{p r i}^{\text {sink }}\right)},\left(K_{\text {pri }}^{k}\right)_{\text {signed }\left(K_{p r i}^{\text {sink }}\right)}\right),
$$

where $\left(K_{p u b}^{k}, K_{p r i}^{k}\right)$ is a newly generated pair of cryptographic keys for token $k$ at a reconfiguration epoch. The reconfiguration protocol is the same as RESIST-1, except that, at the reception of a new epoch and before choosing a sensor $Y$ as its parent, a sensor $X$ challenges the sensor $Y$ first by sending a Challenge $(k$, epoch $)$ message. Basically, this message asks $Y$ to prove its distance $k$ from the sink (i.e. that it has a copy of the token $T_{k}$ ). Sensor $Y$ replies with a message ChallengeReply, which contains:

$$
\left(\left(k, \text { epoch }, K_{p u b}^{k}\right)_{\operatorname{signed}\left(K_{p r i}^{\text {sink }}\right)},\left(I D^{Y}, I D^{X}\right)_{\operatorname{signed}\left(K_{p r i}^{k}\right)}\right)
$$

$\left(k, \text { epoch }, K_{p u b}^{k}\right)_{\operatorname{signed}\left(K_{p r i}^{\operatorname{sink}}\right)}$ is the first half of the token $T_{k}$ that $Y$ received. At the reception of the ChallengeReply message and using the public key $K_{p u b}^{\text {sink }}$, node $X$ can first verify if the token $k$ was correctly signed by the sink. In addition, node $X$ recovers the public key of the token $k, K_{p u b}^{k}$. Then, it can decrypt the second part of the ChallengeReply message, which was encrypted by the private key of token $k, K_{p r i}^{k}$. Node $X$ can thus verify if its identity, $I D^{X}$, was correctly signed by node $Y$. If so, it believes in $I D^{Y}$ and in the $Y$ 's advertised distance $k$ from the sink.

Sinkhole attack resilience: Since a sensor can sign the second part of the ChallengeReply message, if and only if it knows the private key for the token $k$, it is impossible for a compromised sensor (without collusion) to correctly reply to a Challenge. Furthermore, compromised nodes cannot even carry out the attack that we described for RESIST1. Essentially, not dropping the smallest token would fail, because they would not be able to respond to the Challenge for the shorter hop count. Hence, RESIST-0 provides strong resilience against sinkhole attacks. We discuss the impact of collusion on our protocols in Section V.

\section{Performance Evaluation}

The goal of our evaluation is to measure the amount of resilience obtained by RESIST protocols described in Section III. To this end, we present performance results under malicious attacks using three baseline routing protocols, described hereafter. The experiments were run in a discrete event-based simulator implemented in Java. As we are only interested in a RESIST's algorithmic evaluation, our simulator uses a simplified MAC layer, where neither message losses, nor collisions are considered. The correct performance of the presented schemes are independent of the order of message arrival. Furthermore, Hello message losses are typically handled by tree generation algorithms. On the other hand, lost Challenge or ChallengeReply might delay tree generation but do not jeopardize resilience.

\section{A. Simulation Setup}

This section describes the three baseline protocols and our simulation setup. We consider a data collection application, where each sensor only sends data (e.g., measurements) to the sink. The routing topology to reach the sink is regularly reconfigured [10]. Malicious nodes do not generate data and they drop every received message with probability $p=1$.

In our simulator, we implemented three baseline routing protocols: FTree, RRobin and RWalk. We studied the performance of these protocols in networks when resilient reconfiguration schemes are used (RESIST-1 and RESIST-0) and not used (vulnerable case). In addition, compromised nodes try to attract higher volumes of traffic by advertising shorter paths.

In FTree, the routing tree is built once at each topology reconfiguration phase. RRobin differs from FTree as each sensor computes a set of alternative parents during the topology reconfiguration. This set includes the neighbor that sent the first Hello message and any neighbor that sent a Hello message with a hop count smaller or equal to the first neighbor. Each time a sensor has to send a message, it selects one parent from this set in a round robin way. In RWalk protocol, each sensor makes a random decision about forwarding a message either over the routing tree (computed as in FTree) or 


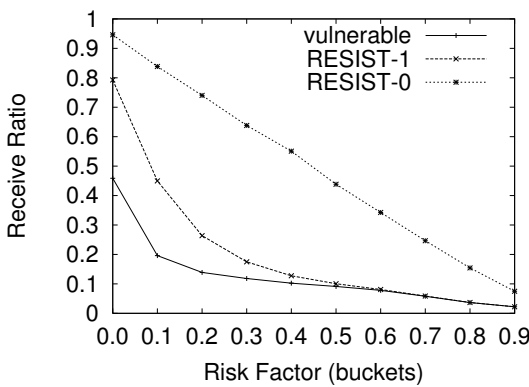

(a) FTree routing

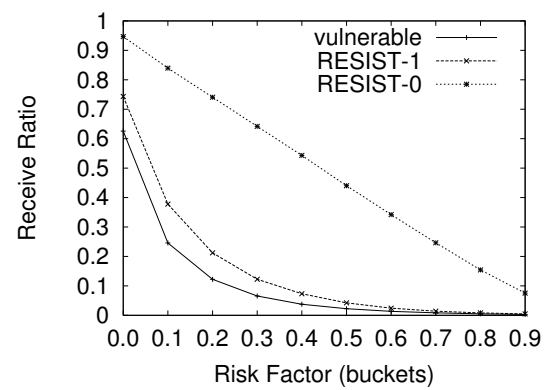

(b) RRobin routing

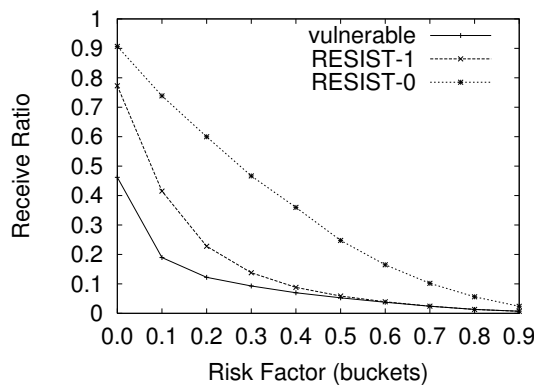

(c) RWalk routing

Fig. 2. Performance gain for different routing protocols: (a) Fixed Tree, (b) Round-Robin with, (c) Random-Walk $(n=1)$

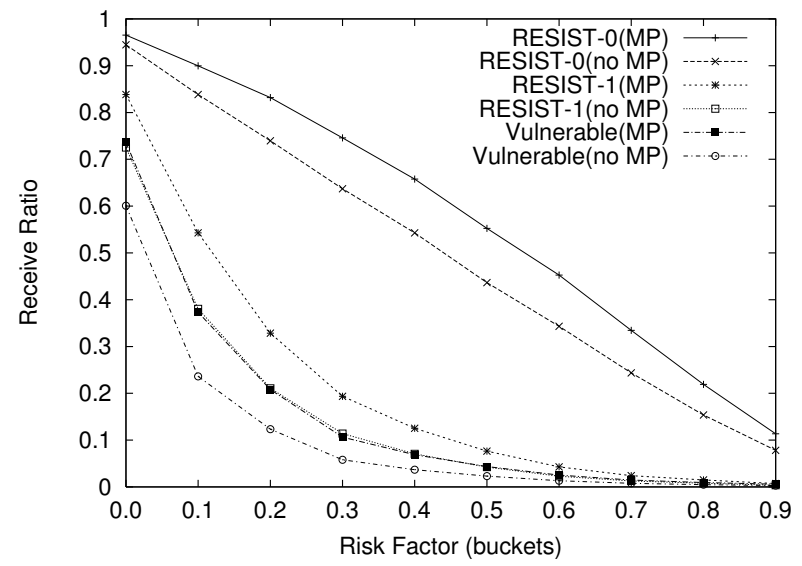

Fig. 3. Using two paths (MP) instead of one increases the overall performance of the different strategies.

forwarding it to a randomly selected neighbor. If the message is not sent over the tree, it follows a $n$-hop random walk and after $n$ hops, it is again forwarded over the tree. The goal of both RRobin and $R$ Walk protocols is to allow escaping regions that may be severely affected by malicious nodes. In all our experiments with $R$ Walk, we use $n=1$.

We generated many random topologies, progressively filling them with malicious sensors. The space of topologies was divided in 10 buckets, where buckets 0 , 1, etc. contain the topologies whose risk factor is respectively in $[0,0.1),[0.1$, 0.2 ), etc. At each step, the risk factor was evaluated and the topology added to the corresponding bucket, until every bucket had at least 100 topologies. Using these topologies, the performance gain was computed as the ratio of messages that actually reach the sink compared to the number of messages that should reach the sink if no sensor were compromised.

\section{B. Resilient Reconfiguration Protocols}

Our results show that RESIST-0 achieves significant performance gain for all routing protocols (see Fig. 2). For both vulnerable and RESIST- 1 cases, the decrease of the receive ratio is roughly exponential, whereas for RESIST-0, it has a better, linear decrease, as it does not allow nodes to lie about their distance to the sink. Fig. 2 confirms that when malicious sensors are able to lie, they can attract more network traffic and thus, incur a much higher impact in the WSN. The linear decrease in performance of RESIST- 0 seems to be the upper bound of the performance we can obtain by only addressing the sinkhole attacks. To get better results, one must also fight selective forwarding attacks. An attractive approach to decrease the impact of selective-forwarding attacks is to send each message through multiple paths to the sink. However, Fig. 3 shows that the improvement from multi-path routing is limited. In our simulations, the performance improvements were limited to $5 \%$ to $10 \%$ when two paths per message was used (one FTree path and another RRobin path), for different resiliency levels. In both RESIST-1 and the vulnerable cases, using more than two paths would not be sufficient to reach the performance of RESIST- 0 .

\section{Routing Protocols Comparison}

We next focus on comparing the performance of the three routing protocols using RESIST-0 (see Fig. 4(a)). Note that even if sinkhole attacks are avoided, malicious nodes can still perform selective forwarding attacks. Fig. 4(a) clearly shows that FTree and RRobin outperform RWalk. This is expected as in $R$ Walk, the average path length that each message travels to the sink is longer. This consequently increases the probability of meeting a malicious node on the path. Further experimentation on RWalk also showed that the protocol performance is inversely proportional to $n$. This actually means that the best case for $n$-hop random walk is achieved when $n=0$, in which case $R$ Walk is equivalent to FTree routing.

Fig. 4(a) also shows that FTree and RRobin have similar performance. This is surprising since, intuitively, the performance of RRobin should be better compared to FTree. Analyzing the results, we observe that, as expected, for sensors, which have malicious parents, RRobin improves the performance by letting these nodes periodically send to alternative parents. However, this does not necessarily improve overall performance as the reverse case also holds: sensor nodes with good parents on the routing tree use malicious nodes as parents in a round robin fashion. Consequently, any gain from RRobin is neutralized by putting sensor nodes with good parents at risk.

To understand the effect of malicious nodes on the protocol behavior better, we divide the network into 100 equal zones and define the failure threshold of a zone as the percentage of data sent by the zone that needs to be dropped to qualify 


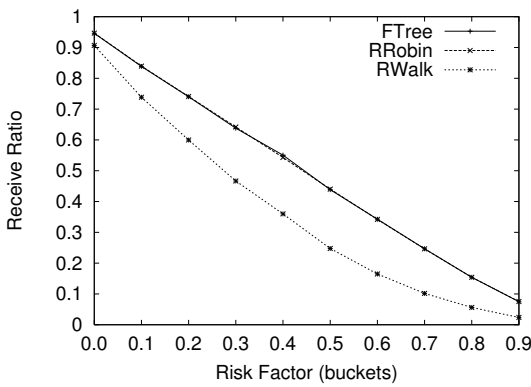

(a)

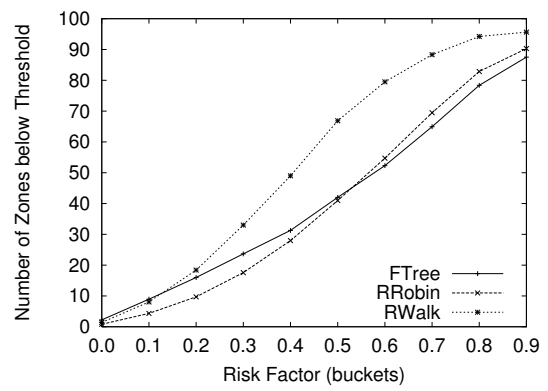

(b)

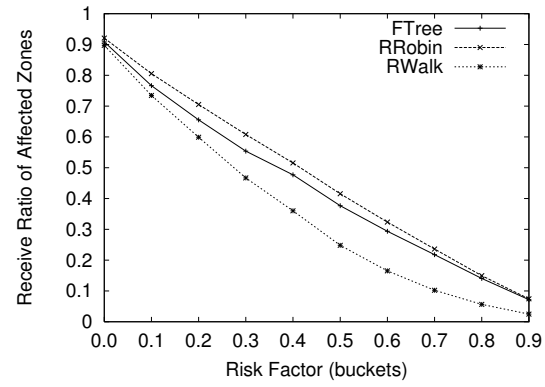

(c)

Fig. 4. RESIST-0 performance evaluation: (a) Comparative performance of routing protocols. (b) Number of severely affected zones in the network (threshold $=60 \%$ ). (c) Received ratio of affected zones in the network.

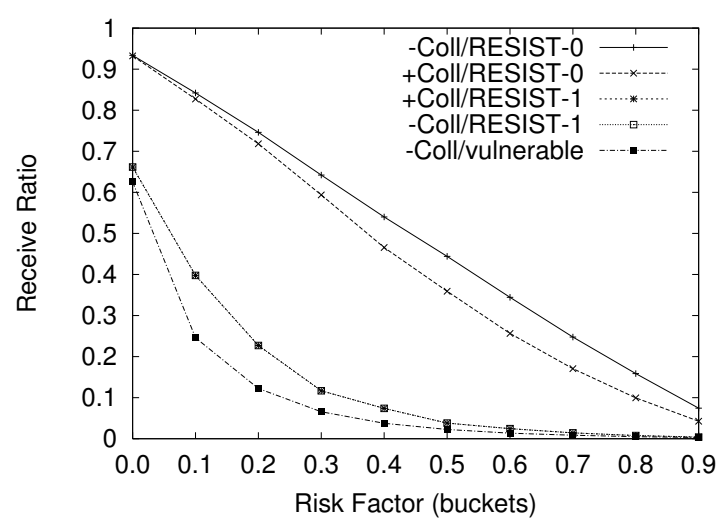

Fig. 5. In the presence of short range collusion (+Coll), RESIST-0 still performs much better than vulnerable routing without collusion. RESIST-1 is not affected, since the default malicious behavior is similar to collusion.

the zone as poorly monitored. In reality, this threshold would depend on the criticality of the sensor network application. We set the failure threshold as $60 \%$ in our experiments. Fig. 4(b) illustrates how many zones fell below the failure threshold for each risk factor bucket and routing protocol. Initially, the number of zones below failure threshold is higher for FTree than RRobin. Coupled with the fact that both protocols share the same receive ratio (see Fig. 4(c)), this means that RRobin just diffuses the effect of malicious nodes to more zones, so that fewer zones actually fail. However, as the risk factor increases, the number of zones below threshold increases beyond FTree due to the reverse case appearing more often. Essentially, increasing the failure threshold moves the shift point to the right. Nevertheless, although the number of affected zones is higher for RRobin, the average received data ratio per affected zone still remains higher than FTree. On the other hand, the number of failed zones in RWalk is always the highest due to its overall poor performance.

\section{Discussion}

We discuss here the cost of using cryptographic primitives in WSNs and the impact of collusion on RESIST protocols.

\section{A. Reducing the Cost of Cryptography for Sensors}

The main cost of our RESIST protocols is the size of Hello messages, which carry multiple tokens that contain cryptographic values. Although, this might strain the significantly resource-constrained WSNs, we believe this problem can be avoided by an efficient way of implementing tokens. First, Elliptic Curves Cryptography (ECC) keys can be used to reduce the size of messages. Signatures and keys of length 110 bits would be sufficient, since ECC keys are as efficient as much longer RSA keys. Recently, [13] shows that ECC can be implemented at a very low cost in WSN.

Second, in [16] one-way hash functions are used so that a token $T_{k+1}$ in RESIST-1 can be computed from a token $T_{k}$. Using this method, Hello messages need only to contain the first and the last token, signed by the sink, i.e. Hello $\left(H^{k}\left(T_{0}\right),\left(\text { epoch }, H^{D}\left(T_{0}\right)\right)_{\text {signed }\left(K_{\text {pri }}^{\text {sink }}\right)}\right)$, where $d$ is the diameter of the network. On receiving this message, a sensor can find its hop-count distance $k$ by hashing $D-k$ times the token $H^{k}\left(T_{0}\right)$ until it reaches $H^{D}\left(T_{0}\right)$. Moreover, as it does not know $T_{0}$, and $H$ is a one-way function, it cannot compute $H^{k-1}\left(T_{0}\right)$. The same idea could be used for RESIST-0: in this case, the public keys $K_{p u b}^{k}$ and $K_{p u b}^{D}$ only are propagated. $K_{p u b}^{k}$ is used to verify the challenge, and also to generate the new ECC key $K_{p r i}^{k+1}$ (with the cost of a modulo operation), from which the public key $K_{p u b}^{k+1}$ is then generated (with the cost of an exponentiation operation), and sent to the neighbours. The chain can be verified by iterating these two operations until $K_{p u b}^{D}$ is reached.

\section{B. The Impact of Collusion on RESIST Protocols}

To be able to implement sinkhole attacks in the presence of RESIST protocols, malicious sensors need to collude, to share good tokens (i.e., a token that can prove a short distance to the sink) they are able to collect during reconfigurations. Thus, RESIST protocols limit the power of collusion attacks to the closest distance an attacker can get to the sink. Hence, malicious nodes residing at the border of the network, as in our initial scenario in the introduction, would not be able to disrupt it.

Collusion is also limited by the communication capabilities of malicious sensors: in Fig. 5, we simulated the impact of collusion when colluding sensors have normal radio ranges and are distributed randomly on the network area. In our simulations, malicious nodes exchange tokens so that they all appear the same distance to the sink (i.e., the distance of the malicious node that is closest to the sink). Our results 
show that the performance of RESIST-1 is not affected by the presence of colluding nodes. This was expected, as the collusion among malicious nodes do not necessarily create a higher impact on the structure of the tree. On the other hand, in RESIST-0, sharing of tokens enables replying challenges for shorter distances and hence, has an effect on performance.

Finally, the most dreadful attack would be a malicious sensor, close to the sink with a long radio range, allowing it to propagate a very good token to malicious sensors far from the sink. However, collusion in this case might not cause a significantly higher degradation in performance, as the dominant impact already comes from the malicious node closest to the sink.

\section{RELATED WORK}

Security has been attracting the attention of many researchers [17], since it is vital to guarantee correct operation of sensor protocols. The main conclusion of recent studies on secure routing is that updating current protocols with security extensions is not sufficient and that routing protocols should be designed from scratch with security in mind.

This paper focuses particularly on sinkhole and selective forwarding attacks. Most other approaches against these attacks revolves around detection of malicious nodes [1], [2], [16]: multi-hop acknowledgments are used in [2], to detect and blacklist nodes that perform selective forwarding attacks. However, in addition to its cost, the proposed scheme requires geographical location information and strict synchronization. In [1], a learning technique based on neural networks is used to predict the sensor measurements, and a reputation scheme is used to mark nodes as faulty if their reports are too different from predictions. In [16], a protocol similar to RESIST-1 is proposed, but without strong cryptography. As a consequence, it requires an additional protocol to detect malicious sensors (reports are vulnerable to falsification) and to blacklist nodes (through a complex messaging mechanism).

An interesting analysis of DDoS attacks in sensor networks, which also takes into account different network parameters and some counter measures, is presented in [18]. While their work covers TCP JellyFish and selective-forwarding attacks, we focus on sinkhole attacks. Moreover, our Risk Factor metric captures more network characteristics.

An intuitive approach against selective forwarding attacks is to use multipath routing [19], [20]. However, such a protocol dramatically increases communication overhead as the redundancy of paths increases. In addition, these paths eventually converge to a few nodes surrounding the base station where malicious nodes can have a dreadful impact. Indeed, our simulation results show that the efficiency of this approach is limited, as confirmed by [18].

Trust-based systems [21], [22] are interesting approaches to deal with selective forwarding attacks. In these systems, interactions between sensors are used for trust level computation. Such systems are, however, often complex. We believe resilience, as provided by our protocols, is a better choice. As in [23], RESIST could use trust levels in the choice of the set of nodes to be considered during the round robin procedure.

\section{CONCLUSION}

We presented two cryptographic reconfiguration protocols that increase the resilience of the network in the presence of sinkhole attacks: RESIST-1 prevents malicious nodes from changing their advertised distance to the sink more than one hop; RESIST-0 completely stops malicious nodes from lying about their distance, but is more expensive to use. Our performance evaluation confirmed the higher resilience of our protocols, even in the presence of some collusion for three basic routing protocols. We discussed the implementation issues of our protocols, showing that Elliptic Curves Cryptography (ECC) provides an interesting way to encode the protocol in significantly short messages. Finally, collusion between compromised sensors, even if possible, has limited impact on the real performance of our protocols.

\section{REFERENCES}

[1] P. Mukherjee and S. Sen, "Using learned data patterns to detect malicious nodes in sensor networks," in ICDCN, 2008.

[2] B. Yu and B. Xao, "Detecting selective forwarding attacks in wireless sensor networks," in IEEE IPDPS, 2006.

[3] N. Ramanathan, K. Chang, R. Kapur, L. Girod, E. Kohler, and D. Estrin "Sympathy for the sensor network debugger," in Sensys, Nov. 2005.

[4] C. Intanagonwiwat, R. Govindan, and D. Estrin, "Directed diffusion: a scalable and robust communication paradigm for sensor networks," in ACM MOBICOM, 2000.

[5] F. Ye, A. Chen, S. Lu, and L. Zhang, "Gradient broadcast: A robust, long-live large sensor network," UCLA, Tech. Rep., 2001.

[6] — "A scalable solution to minimum cost forwarding in large sensor networks," in Conf. on Computer Communications and Networks, 2001.

[7] U. Cetintemel, A. Flinders, and Y. Sun, "Power-efficient data dissemination in wireless sensor networks," in ACM MobiDE, 2003.

[8] B. Krishnamachari, D. Estrin, and S. Wicker, "The impact of data aggregation in wireless sensor networks," in IEEE ICDCS, 2002.

[9] Y. J. Zhao, R. Govindan, and D. Estrin, "Residual energy scans for monitoring wireless sensor networks," IEEE WCNC, 2002.

[10] S. Madden, M. J. Franklin, J. M. Hellerstein, and W. Hong, "TAG: a Tiny Aggregation Service for Ad-Hoc Sensor Networks," OSR, 2002.

[11] L. B. Oliveira, D. Aranha, E. Morais, F. Daguano, J. Lpez, and R. Dahab, "Identity-based encryption for sensor networks," in PERCOM, 2007.

[12] S. A. amtepe and B. Yener, "Key distribution mechanisms for wireless sensor networks: a survey," IEEE/ACM Trans. on Networking, 2005.

[13] D. J. Malan, M. Welsh, and M. D. Smith, "Implementing public-key infrastructure for sensor networks," ACM Trans. Sen. Netw., 2008.

[14] A. Papadimitriou, F. Le Fessant, A. C. Viana, and C. Sengul, "Fighting sinkhole attacks in tree-based routing topologies," INRIA, Tech. Rep. RR-6811, 2008.

[15] F. Le Fessant, C. Sengul, and A.-M. Kermarrec, "Pace-maker: Tracking peer availability in large networks," INRIA, Tech. Rep. RR-6594, 2008.

[16] S.-B. Lee and Y.-H. Choi, "A secure alternate path routing in sensor networks," Computer Communications, Elsevier, vol. 30, 2006.

[17] W. Yu and K. Liu, "Attack-resistant cooperation stimulation in autonomous ad hoc networks," IEEE/ACM Trans. on Networking, 2005.

[18] I. Aad, J.-P. Hubaux, and E. W. Knightly, "Impact of denial of service attacks on ad hoc networks," IEEE/ACM Trans. on Networking, 2008.

[19] C. Karlof and D. Wagner, "Secure routing in wireless sensor networks: Attacks and countermeasures," in 1st IEEE Workshop on Sensor Network Protocols and Applications (SNPA), 2003.

[20] S. M. Jing Deng, Richard Han, "Insens: Intrusion-tolerant routing in wireless sensor networks," in IEEE ICDCS, 2003.

[21] S. Marti, T. Giuli, K. Lai, and M. Baker, "Mitigating routing misbehavior in mobile ad hoc networks," in ACM MOBICOM, 2000.

[22] Y. Sun, Z. Han, W. Yu, and K. J. R. Liu, "A trust evaluation framework in distributed networks: Vulnerability analysis and defense against attacks," in IEEE INFOCOM, 2007

[23] Z. Liu, A. Joy, and R. Thompson, "A dynamic trust model for mobile ad hoc networks," in Future Trends of Dist. Computing Systems, 2004. 